\title{
Les écoles confessionnelles en Europe
}

Les raisons d'un succès

Religious schools in Europe. The reasons for their success

Las escuelas confesionales en Europa. Las razones de un éxito

Michael S. Merry

Traducteur : Eva Loechner

\section{(2) OpenEdition}

\section{Journals}

Édition électronique

URL : https://journals.openedition.org/ries/6062

DOI : 10.4000/ries.6062

ISSN : 2261-4265

Éditeur

France Education international

Édition imprimée

Date de publication : 1 décembre 2017

Pagination : 73-82

ISBN : 978-2-85420-616-6

ISSN : $1254-4590$

Référence électronique

Michael S. Merry, "Les écoles confessionnelles en Europe », Revue internationale d'éducation de Sèvres

[En ligne], 76 | décembre 2017, mis en ligne le 01 décembre 2019, consulté le 25 juin 2021. URL http://journals.openedition.org/ries/6062 ; DOI : https://doi.org/10.4000/ries.6062 


\title{
Les écoles confessionnelles en Europe
}

\section{Les raisons d'un succès}

\author{
Michael S. Merry \\ Université d'Amsterdam
}

En raison du grand nombre de pays européens et de la diversité des systèmes de gouvernement entre ces pays et en leur sein, quiconque se penche sur la question des écoles confessionnelles en Europe se doit d'examiner chaque contexte dans sa spécificité. À travers le continent européen, des différences considérables se rencontrent, s'agissant des aspects suivants : le christianisme, une dénomination historiquement dominante ; la liberté d'opérer accordée aux écoles confessionnelles, sur le plan institutionnel mais également associatif, pédagogique et organisationnel; les systèmes de reddition de comptes mis en place; les tendances conservatrices ou progressistes de chaque école prise individuellement ; les significations différentes revêtues par les termes "public » et "privé " dans le domaine éducatif ; le pourcentage d'enfants fréquentant des écoles confessionnelles ; le choix de privilégier ou non, à l'embauche, des membres d'une confession donnée; le degré de concurrence créé par la «loi du marché » entre les écoles confessionnelles et leurs équivalents laïcs, etc. Ces facteurs, et de nombreux autres, influencent la façon dont les écoles confessionnelles continuent de fonctionner en Europe au vingt-et-unième siècle.

Cependant, étant donné la longue tradition d'imbrication de l'Église et de l'État dans le contexte européen, il peut être utile d'envisager ces différences en termes de financement et de gestion par l'État (Glenn et de Groof, 2002). Il est important de se pencher brièvement sur cette question du financement public, compte tenu de l'impact qu'a l'engagement de l'État sur la gestion des écoles, les programmes qu'elles doivent appliquer, les types d'instruction religieuse qu'elles proposent, les attentes académiques lors des examens, les types d'examens mis en place, et bon nombre d'autres aspects. Sur cette question, le tableau est très contrasté : dans certains pays, comme la Bulgarie, il n'existe aucun financement d'État pour les écoles confessionnelles. Dans d'autres, comme l'Espagne et la France, le financement public dépend d'une relation contractuelle avec l'État, et ce contrat favorise en général la confession historiquement dominante. Ailleurs, comme en Scandinavie, le financement de l'État est généreux mais seulement partiel : il couvre environ les trois quarts du coût de fonctionnement des écoles. Dans un pays comme l'Allemagne, le financement public varie en fonction des

* Article traduit par Eva Loechner. 
Länder mais s'échelonne entre 55 et $85 \%$. Les Pays-Bas sont peut-être le seul pays qui finance à $100 \%$ ses écoles, qu'elles soient confessionnelles ou non, à condition qu'elles remplissent un cahier des charges précis. En fonction du type d'élèves qu'elles accueillent, il arrive même que certaines écoles confessionnelles reçoivent davantage d'aides de l'État que les écoles laïques, afin de compenser le handicap socio-économique qui pèse sur leur public.

\section{ÉVOLUTIONS RÉCENTES}

Depuis le début des années 1970, certains changements importants sont survenus en Europe (à un degré plus ou moins grand selon les pays) et bon nombre de ces changements ont eu des conséquences dans le domaine éducatif. Tout d'abord, avec l'arrivée massive de travailleurs immigrés issus de groupes ethniques et de religions différents après la Seconde Guerre mondiale, et en particulier depuis le milieu des années 1960, les pays européens ont été confrontés à la difficulté de faire face à cette diversité inédite. Chaque pays a sa propre façon de s'adapter à cette diversité religieuse : en Grèce par exemple, cette "diversité " renvoie à toute école autre qu'une école publique imprégnée des valeurs de la religion grecque orthodoxe ; en Irlande et en Belgique, toute école autre que catholique; et, jusqu'à très récemment, dans de nombreux pays du nord de l'Europe, toute école confessionnelle autre que luthérienne ou réformée.

Même si l'on rencontrait déjà des écoles juives financées par l'État en Europe dès les années 1950, la reconnaissance officielle des "nouvelles religions » (c'est-à-dire l'hindouisme, le bouddhisme, le sikhisme, l'islam) a commencé au début des années 1970. À partir des années 1980, de timides tentatives ont été faites dans de nombreux pays pour modifier les programmes (souvent de façon purement symbolique), afin de "reconnaître " la forte présence d'enfants issus de minorités dans les écoles. Parfois, comme en Belgique, des efforts (modestes) ont été entrepris afin de proposer une instruction religieuse aux confessions non chrétiennes, l'islam en particulier. Ensuite, à la fin des années 1980, les pays européens ont peu à peu pris conscience que ces populations immigrées (et musulmanes, principalement) s'étaient installées chez eux de façon plus ou moins permanente. C'est à la fin de cette décennie que, dans certains pays européens, les premières écoles musulmanes financées par l'État sont apparues. À la fin des années 1990, plusieurs écoles hindouistes ont été créées aux Pays-Bas. En Angleterre, au début des années 2000, la création d'un petit nombre d'écoles sikhes et hindouistes a également coïncidé avec l'expansion des écoles confessionnelles plus généralement, sous le gouvernement New Labour de Tony Blair.

Ces évolutions ont été concomitantes de la rapide montée de mouvements populistes d'extrême-droite ouvertement hostiles à l'immigration (et, la plupart du temps, hostiles à l'islam également). Avec leurs inquiétudes vis-à-vis $\mathrm{du}$ communautarisme ou $\mathrm{du}$ " choc des civilisations », ces partis politiques ont attisé des sentiments xénophobes et islamophobes latents et obtenu un succès 
considérable auprès de populations préoccupées par ces changements démographiques et un climat économique morose. Au sein des partis politiques plus traditionnels, l'urgence de mettre en place des "politiques d'intégration» et les inquiétudes explicites vis-à-vis de minorités faisant l'objet de ségrégations sont également devenus des thèmes incontournables. Les écoles confessionnelles destinées à des minorités - et en particulier les écoles musulmanes - ont été pointées du doigt comme des preuves de «l'échec de l'intégration» (Maussen et Bader, 2015).

Enfin, au début des années 1990, une série de mesures néo-libérales est venue transformer les systèmes éducatifs de nombreux pays européens. Nombre de ces réformes ont été présentées comme des façons d'étendre la liberté de choix des parents et l'autonomie des établissements. En effet, des tentatives ont été faites pour "décentraliser » le plus possible la chaîne du pouvoir à l'échelon local. Pourtant, dans le même temps, des directives plus strictes et plus autoritaires sont devenues de plus en plus la norme. En parallèle de ces réformes, une nouvelle forme de compétition entre établissements est apparue, qui a coïncidé avec l'émergence de classements des établissements scolaires et a été accélérée par le développement de réseaux de parents d'élèves mieux éduqués et informés. Désireux de trouver de "meilleures » écoles pour leurs enfants, nombre d'entre eux se sont rapidement révélés aussi doués pour s'orienter parmi une offre scolaire pléthorique et déroutante que pour faire en sorte que leurs enfants disposent des moyens de transport nécessaires pour se rendre dans ces écoles. De leur côté, les établissements scolaires ont réagi à ces évolutions en se "vendant» de plus en plus aux parents, allant parfois jusqu'à s'inventer une identité de niche, afin de se distinguer de leurs concurrents (Maussen et Bader, 2015). Pourtant, même si de nombreux pays disposent de systèmes éducatifs offrant des choix très variés (comme, par exemple, les «écoles libres » danoises), la décision de se tourner vers une école confessionnelle peut laisser perplexe, à une époque où la pratique religieuse a diminué de façon spectaculaire depuis la fin des années 1960. Comment, dès lors, expliquer ce choix ? Ci-dessous sont présentées plusieurs explications plausibles.

\section{LES RAISONS DE CHOISIR UNE ÉCOLE CONFESSIONNELLE}

Il est évident que la plupart des gens préfèrent côtoyer et rencontrer des personnes qui leur ressemblent. Ces préférences rendent possibles des modes d'identification et d'interaction fondés sur des expériences, des habitudes et des intérêts partagés. Nous reconnaissons là les caractéristiques de l'association volontaire, qui influence profondément à la fois les choix résidentiels et les habitudes de socialisation. L'association volontaire influe également directement sur les motivations des parents dans leur choix d'école pour leur enfant. Toutefois, étant donné que la géographie sociale reflète déjà une forme d'association 
volontaire, il se peut que les motivations parentales résultent moins d'une intention consciente que d'un choix " évident ", lié à l'environnement dans lequel ils vivent. Examinons à présent trois raisons de choisir une école confessionnelle qui sont largement vérifiées de façon empirique.

\section{La proximité}

Choisir une école proche de chez soi, qu'elle soit confessionnelle ou non, constitue une des raisons pour lesquelles les écoles confessionnelles demeurent aussi populaires, en particulier au sein de communautés plus restreintes dans lesquelles les écoles confessionnelles ont longtemps représenté le seul choix possible. L'Angleterre est un bon exemple, puisque les écoles anglicanes et catholiques existaient bien avant que l'État ne prenne en charge l'éducation des enfants. Mais dans de nombreux pays européens, la quasi-omniprésence des écoles confessionnelles signifie qu'elles sont tout simplement familières pour les parents et constituent donc le choix par défaut. À cela s'ajoute qu'il y a rarement de différence entre les écoles confessionnelles et laïques en termes de contenu académique ou de valeur des diplômes. Lorsque, en particulier, ce sont les parents qui se chargent d'amener les enfants à l'école, et lorsque des emplois du temps surchargés ne permettent pas de se tourner vers une école différente, la distance entre l'école et le domicile est un critère très important. Les écoles confessionnelles peuvent en général répondre à ce besoin aussi bien que toute autre. Et comme de nombreux pays européens ont en commun un passé de ségrégation religieuse - principalement entre les confessions protestante, catholique et orthodoxe - de nombreux quartiers et leurs écoles portent toujours les traces de cette histoire. Dès lors, dans de nombreux pays, les écoles publiques sont en réalité des écoles confessionnelles (par exemple les écoles imprégnées de l'ethos orthodoxe en Grèce et en Serbie ou de l'ethos catholique en Bavière, en Autriche et en Irlande). Cependant, du moment que l'école est perçue comme faisant partie intégrante de la communauté et que sa qualité est acceptable, son caractère confessionnel ne pose pas de problème pour de nombreux parents.

\section{LA FOI}

Il ne faudrait pas négliger les motivations explicitement religieuses. Des parents très pratiquants qui souhaitent renforcer leur propre attachement à la religion choisissent souvent une école confessionnelle. Dans de nombreux pays européens, une minorité distincte d'écoles juives, chrétiennes ou musulmanes conservatrices ont réussi à se positionner comme une alternative aux écoles qui n'ont de confessionnel que le nom. Les juifs, musulmans, catholiques et protestants très pratiquants (chez ces derniers, surtout ceux qui appartiennent aux églises réformées et évangéliques) font de l'authenticité des valeurs religieuses d'une école l'une de leurs principales priorités. Les écoles confessionnelles qui s'adressent à ce public de niche peuvent se rendre plus attractives auprès des 
parents en mettant en avant l'aspect central de la foi (par exemple, la lecture des écritures, la prière du vendredi, les célébrations liturgiques) mais également en perpétuant des pratiques traditionnelles (comme la non-mixité ou des règles concernant l'habillement des élèves) que de nombreux parents pratiquants prisent tout particulièrement. Les écoles confessionnelles conservatrices s'efforcent d'incorporer la doctrine religieuse dans toutes les disciplines, y compris les sciences, que beaucoup considèrent comme étant en désaccord avec leur foi (par exemple la théorie darwinienne de l'évolution). Être capable de choisir une école authentiquement confessionnelle devient ainsi une marque distinctive par rapport à d'autres écoles qui ne le sont que nominalement.

\section{LA RÉPUTATION ACADÉMIQUE}

Une troisième explication au choix d'une école confessionnelle tient à la réputation académique de l'établissement. Lors de leur analyse des données de l'OCDE, Dronkers et Avram (2015) différencient plusieurs «stratégies de choix » en Europe. Selon eux, un milieu social aisé et des moyens financiers importants constituent la raison principale de choisir ce qu'ils appellent " des écoles ne dépendant pas de l'État " dans un petit groupe de pays, où ces écoles s'adressent en particulier aux enfants issus de familles de la classe supérieure. Pour le reformuler plus crûment, on pourrait dire que des parents au capital social plus élevé sont susceptibles d'agir de la même façon lorsqu'il s'agit de choisir une école, et le fait de se tourner vers des écoles permettant aux enfants de se distinguer des "enfants des autres » est un comportement régulièrement documenté dans les études de sociologie de l'éducation (Ball, 2002).

Afin de s'assurer de la réputation académique d'une école, certains parents peuvent consulter les résultats aux examens ou les classements des établissements disponibles dans les médias, ou encore les rapports officiels d'inspection publiés sur Internet, mais pour la plupart des parents il est en général assez difficile de déterminer la qualité d'une école en particulier, en dehors de quelques critères évidents, comme le type de public qui la fréquente. C'est pourquoi de nombreux parents n'ont d'autre solution que de se fier au bouche-à-oreille - c'est-à-dire à l'opinion de gens qui leur ressemblent - ou à la " marque » de l'école. Toutefois, la capacité d'une école confessionnelle à conserver une bonne réputation académique semble en réalité dépendre moins de sa qualité intrinsèque que du fait que s'y rassemblent des personnes issues d'un milieu social identique, ainsi que le suggèrent Dronkers et Avram. Dès lors, une école « meilleure » n'est peut-être, en réalité, qu'une école fréquentée par un public plus homogène d'élèves issus de milieux favorisés.

Il n'est évidemment pas toujours vrai que les écoles confessionnelles sont de meilleure qualité que les autres. La qualité d'une école dépend de toute façon d'une grande variété de variables en fonction des circonstances. De nombreuses données suggèrent toutefois que beaucoup d'écoles confessionnelles 
offrent une éducation de meilleure qualité que leurs équivalents laïcs en termes de "valeur ajoutée » à l'apprentissage des élèves. Ces études indiquent que beaucoup de ces écoles mettent l'accent sur le maintien de bonnes relations informelles entre l'administration et le corps enseignant, ce qui contribue à un meilleur climat scolaire, un aspect souvent avantageux pour des élèves issus de minorités défavorisées (Driessen, Agirdag et Merry, 2016). Nombre d'entre elles, de fait, parviennent bien mieux à maintenir une gouvernance efficace, des effectifs moins lourds, une discipline plus stricte et de meilleurs résultats. Ces études font également l'objet de critiques, notamment en raison du fait que les écoles confessionnelles se réservent souvent le droit d'exercer un certain degré de sélection, qu'il s'agisse du recrutement des enseignants ou de l'admission des élèves. Des procédures disciplinaires peuvent également avoir pour résultat d'exclure des élèves « indésirables ». En outre, même si les écoles confessionnelles financées par l'État ne peuvent exercer ouvertement de discrimination contre des élèves, elles ont, de fait, souvent la possibilité de refuser des enfants handicapés, sous prétexte qu'elles ne possèdent pas les équipements ou le personnel approprié, et ont généralement également la possibilité d'exclure des élèves dont le personnel a du mal à gérer le comportement, sous prétexte qu'ils enfreignent les règles de conduite de l'établissement.

Les lecteurs qui sont familiers de la recherche empirique sur les écoles confessionnelles en Europe (et ailleurs) ne seront probablement pas étonnés par la plupart de ces raisons. Toutefois, ces explications et d'autres du même type ne rendent pas entièrement compte du phénomène. Je crois en effet qu'il existe également d'autres motivations, plus cachées, et qui demeurent pour cette raison insuffisamment étudiées et mal comprises. Dans ce qui suit, je suggère quelques hypothèses au sujet d'autres facteurs rarement explicités - et donc difficilement mesurables - mais toutefois pertinents pour comprendre pourquoi les écoles confessionnelles (et plus particulièrement les écoles protestantes et catholiques) continuent de jouir d'une telle popularité en Europe.

\section{AU-DELÀ DES DONNÉES EMPIRIQUES}

Les variables exposées jusqu'ici ne sont pas les uniques facteurs qui influencent la décision de choisir une école confessionnelle. Et de toute façon, même si les parents expriment certaines raisons d'avoir fait ce choix, si l'on prend en compte les contextes culturels préexistants et les normes institutionnelles - pour ne rien dire du caractère limité des ressources et des opportunités -, leurs choix sont en général très restreints. Même des parents relativement privilégiés peuvent ne pas obtenir leur premier choix d'école pour leur enfant. Mais pour ceux issus de milieux très défavorisés (et discriminés), les options sont souvent encore plus limitées. Il est indéniable que, dans le domaine de l'éducation, les minorités défavorisées et discriminées bénéficient très rarement des mêmes 
options que les membres plus favorisés du groupe dominant. En effet, dans le contexte européen, et tout particulièrement dans les environnements urbains aux populations mélangées, les évolutions démographiques ont rendu plus visibles les phénomènes déjà existants de ségrégation sociale mais également religieuse. Dès lors, dans l'Europe du XXI ${ }^{\mathrm{e}}$ siècle, la ségrégation sociale est désormais souvent renforcée par une ségrégation ethnique et raciale.

Quel rapport tout ceci entretient-il avec le sujet qui nous intéresse, celui des écoles confessionnelles? Tout d'abord, comme nous l'avons vu, de nombreuses écoles confessionnelles, en raison de leurs valeurs fondamentales et de leurs exigences, ont beaucoup moins de difficultés à établir et maintenir un projet éducatif cohérent susceptible d'attirer les professeurs et les parents qui les partagent. En outre, les écoles fondées sur un projet éducatif et une gouvernance solides parviennent souvent à imposer des comportements vertueux, source de davantage de satisfaction pour les professeurs et d'un plus grand bien-être pour les élèves. Le respect, la coopération et l'autodiscipline ont pour conséquence des élèves qui se conduisent mieux et un meilleur climat au sein de l'école, plus difficiles à obtenir dans des écoles où de telles valeurs sont absentes. Ces facteurs non académiques influencent aussi de façon très claire les résultats académiques des élèves (Driessen, Agirdag et Merry, 2016). Ensuite, dans les plus grandes villes européennes, une proportion élevée d'écoles publiques non confessionnelles sont désormais des écoles où les élèves sont en majorité issus de minorités. C'est-à-dire qu'une majorité d'élèves qui fréquentent des écoles publiques "urbaines» sont désormais d'origine non occidentale (autrement dit : non blancs), et les résultats de ces écoles sont inférieurs à ceux d'écoles qui sélectionnent davantage leurs élèves. Et il est important ici de garder à l'esprit le fait que les écoles protestantes et catholiques, même dans des environnements où les minorités sont majoritaires, parviennent souvent à demeurer des bastions pour le groupe indigène dominant. Elles servent donc d'instruments d'exclusion. ${ }^{1}$

Ces mécanismes d'exclusion continuent d'inciter de nombreuses minorités culturelles et religieuses à créer des écoles capables de mieux répondre aux besoins de leurs propres enfants. En effet, même si les écoles protestantes et catholiques en milieu urbain ne fonctionnent pas systématiquement comme des instruments d'exclusion, lorsque c'est le cas, les parents issus de ces minorités religieuses peuvent légitimement estimer que le système scolaire existant ne répond pas aux besoins de leurs enfants. Dès lors, les raisons d'étendre le réseau d'écoles hindoues, coraniques et sikhes se multiplient. Il reste à déterminer si ces «nouvelles» écoles vont tenir leurs promesses, mais les études existantes

\footnotetext{
1. Pour avoir un exemple récent de ce phénomène en Belgique, on pourra consulter un article paru dans De Morgen le 2 septembre 2017, dans lequel on peut lire le récit d'un enseignant dans une « école de concentration » : « maar de [witte] ouders [in de buurt] hebben natuurlijk ook wel gekozen voor een katholieke school... " (mais les parents [blancs] [à proximité] ont bien sûr aussi opté pour une école catholique...). [https://goo.gl/D2FYVX]
} 
(Merry et Driessen, 2012 ; 2016) indiquent de plus en plus clairement que leur valeur ajoutée pour l'éducation d'élèves issus de minorités religieuses est significative et quantifiable.

\section{LA SÉGRÉGATION SCOLAIRE LIÉE À LA RELIGION}

Comme nous l'avons vu, l'association volontaire entre individus semblables tend à entraîner des phénomènes de concentration spatiale - c'està-dire de ségrégation - dans divers domaines, y compris l'école. On ne saurait donc s'étonner que les écoles confessionnelles reproduisent ces phénomènes de ségrégation. Il est probable que cette ségrégation a favorisé la multiplication d'écoles confessionnelles de religions minoritaires, dans lesquelles les parents peuvent exercer leurs droits constitutionnels à choisir une école qui s'adresse à leur communauté culturelle et religieuse. Ce choix est particulièrement séduisant pour des parents qui ont le sentiment que leurs enfants ne sont pas assez stimulés dans le système scolaire, voire y souffrent.

Comme nous venons de l'indiquer, si la ségrégation scolaire créée par des religions minoritaires, comme dans les écoles musulmanes et hindouistes, ne pose pas de problème aux parents qui les choisissent, elles sont souvent perçues avec consternation par de larges pans de l'opinion européenne ${ }^{2}$. Même les défenseurs des droits des minorités, des victimes de discriminations ou des personnes défavorisées sont sceptiques vis-à-vis des écoles confessionnelles destinées à des groupes minoritaires. La raison tient à l'idée, largement partagée, que les écoles confessionnelles conduisent au communautarisme et à la méfiance des groupes les uns envers les autres. C'est ainsi que le philosophe politique canadien Will Kymlicka, un penseur libéral qui a longtemps défendu les droits des minorités, et dont les travaux ont une grande influence sur les chercheurs européens, suggère pourtant que «ce dont les immigrés ont peut-être le plus besoin, ce n'est pas d'écoles séparées, mais d'une approche plus multiculturelle de l'éducation au sein des écoles publiques ouvertes à tous »(2009). En effet, ce sont généralement les écoles confessionnelles destinées à des minorités discriminées ${ }^{3}$ qui sont pointées du doigt par les critiques, tout particulièrement les écoles musulmanes. À la place, dans toute l'Europe, il est de plus en plus question d' « intégration » (Joppke, 2007), alors que les concentrations de populations majoritaires ne sont jamais remises en question. Ces théories intégrationnistes viennent appuyer l'idée que les écoles séparées sont un problème dans une société qui

\footnotetext{
2. De nombreux exemples viennent étayer cette idée. On la trouve par exemple dans les deux articles suivants : « Les écoles confessionnelles fragmentent les communautés " (The Guardian : [https://goo.gl/hacpgo]) ou "Les écoles confessionnelles encourageraient le racisme » (Irish examiner : [https://goo.gl/RSr6hK]).

3. Il existe évidemment d'autres écoles confessionnelles « minoritaires » destinées à des membres de groupes indigènes majoritaires. Il s'agit généralement de confessions chrétiennes comme les Adventistes du septième jour ou les protestants évangéliques.
} 
promeut la tolérance et le multiculturalisme. Il est de loin préférable, selon elles, que des enfants issus de milieux différents soient rassemblés et se concentrent sur ce qu'ils ont en commun : nous discernons là les contours d'une thèse que l'on pourrait appeler "l'école commune».

Il est sans doute vrai que, dans certaines conditions spécifiques, l'éducation "intégrée " commune est bénéfique à tous les enfants. Pourtant, même si chacun s'accordera à dire que l'intégration et l'éducation en commun sont des idéaux tout à fait dignes d'être poursuivis, les « impératifs d'intégration » que certains défendent entraînent en général bien moins de sacrifices pour les membres de groupes majoritaires dont les milieux d'origine correspondent davantage aux habitudes, normes et valeurs institutionnalisées. En effet, les conditions nécessaires à une bonne "intégration " sont souvent difficiles à atteindre, étant donné (a) la nette persistance de mécanismes de ségrégation scolaire, encouragés à la fois par les pratiques normatives d'association volontaire et le soutien légal et constitutionnel apporté à la liberté de choix en matière d'éducation, deux tendances dont rien n'indique qu'elles vont s'inverser; et (b) la manière dont les écoles mettent régulièrement en place des regroupements, des procédures disciplinaires et des labels éducatifs spéciaux qui affectent de façon disproportionnée les élèves pauvres et issus de minorités, d'autant plus, est-on en droit de penser, dans les écoles aux populations "mélangées » (Schmidt et al., 2015).

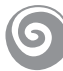

Quels que soient les contextes locaux ou nationaux, les écoles confessionnelles continuent d'être des lieux de contestation. Elles le sont, tout d'abord, car nombreux sont ceux qui leur sont ouvertement hostiles. C'est-à-dire que leur simple existence est considérée comme un scandale par ceux qui estiment que l'éducation devrait être strictement laïque. Mais elles le sont également d'une autre manière, puisque des garanties constitutionnelles ainsi que les forces du marché ont garanti leur survie. En effet, les écoles confessionnelles continuent d'être populaires dans toute l'Europe, en dépit des oppositions, d'une pratique religieuse en fort déclin, et de la croyance persistante que l'Europe est devenue «laïque ». Dans ce qui précède, j'ai essayé de proposer quelques raisons qui expliquent ce succès. Parmi elles, on peut mentionner les aspects pratiques, la réputation académique, les garanties constitutionnelles pour la liberté de choix en matière d'éducation, les phénomènes d'association volontaire, aux conséquences multiples, les mécanismes de ségrégation historiquement ancrés, et la réaction de certains parents à l'exclusion de leurs enfants du système scolaire dominant. Ni les complaintes au sujet de la ségrégation ni les efforts pour promouvoir la «cohésion sociale » et la "citoyenneté » ne semblent réellement à même de mettre en danger la position solide dont les écoles confessionnelles continuent de jouir sur le marché de l'éducation. Les écoles confessionnelles ont, à n'en pas douter, un bel avenir devant elles en Europe. 


\section{BIBLIOGRAPHIE}

BALL S. (2002) : Class Strategies and the Education Market: The Middle Classes and Social Advantage, London : Routledge.

DRIESSEN G., AGIRDAG O., MERRY M.S. (2016) : "The Gross and Net Effects of Primary School Denomination on Pupil Performance in the Netherlands ", Educational Review 68 (4) : 466-480.

DRONKERS J., AVRAM S. (2015) : "What can international comparisons teach us about school choice and non-governmental schools in Europe? ", Comparative Education 51 (1) : 118-132.

GLENN C.L., DE GROOF G. eds. (2002) : Finding the Right Balance: Freedom, autonomy and accountability in education, vol. 1-2, Utrecht : Lemma.

JOPPKE C. (2007) : «Beyond national models: Civic integration policies for immigrants in Western Europe ", West European Politics 30 (1) : 1-22.

KYMLICKA W. (2009) : The Governance of Religious Diversity: the old and the new, in (eds) P. Bramadat et M. Koenig, International Migration and the Governance of Religious Diversity, p. 323-334, Montreal/Kingston : McGill-Queen's University Press.

MAUSSEN M., BADER V. (2015) : « Non-governmental religious schools in Europe: institutional opportunities, associative freedoms and contemporary challenges", Comparative Education 51 (1) : 1-21.

MERRY M.S., DRIESSEN G. (2012) : « Equality on Different Terms: the case of Dutch Hindu Schools ", Education and Urban Society 44 (5) : 632-648.

MERRY M.S., DRIESSEN G. (2016) : «On the Right Track? Islamic Schools in the Netherlands after an Era of Turmoil », Race, Ethnicity and Education 19 (4) : 856-879.

SCHMIDT W. et al. (2015) : "The role of schooling in perpetuating educational inequality: An international perspective », Educational Researcher 44.7 : 371-386. 